\title{
PROIEZIONE OBLIQUA DEL PIEDE, IN RADIOLOGIA CONVENZIONALE, NELLA DIAGNOSI DI TENDINITE DEL PERONIERO BREVE
}

Dott. Prioreschi Tommaso ${ }^{1}$, Dott.sa Rubino Mariele Maria Leondina²

ITSRM USL TOSCANA CENTRO, Ospedale PIOT San Marcello P.se., Pistoia

${ }^{2}$ TSRM Libera professionista, Caserta

KEYWORDS: tendine peroneo breve, radiologia convenzionale, corretta tecnica espositiva/, biomeccanica

\section{ABSTRACT}

L'obiettivo di questo case report è dimostrare che: la connessione tra ascolto e osservazione del paziente, immagini radiografiche guidate dalla biomeccanica e la giusta tecnica espositiva siano fondamentali non solo nello del studio del sistema scheletrico, ma consentano anche l'ottenimento di immagini per lo studio e la diagnosi di patologie a carico del sistema muscolare scheletrico.

\section{INTRODUZIONE}

Muscolo e tendine peroneo breve

Il muscolo peroneo breve origina dal terzo medio della faccia laterale della fibula, $i$ fasci decorrono verticali in basso continuando nel tendine omonimo; è innervato dal nervo peroniero superficiale (L5/S1) e contraendosi abduce e ruota esternamente il piede. Il tendine peroniero breve è piuttosto appiattito e origina dal muscolo, anch'esso piatto, che decorre più profondamente al peroniero lungo, come possiamo osservare in figura 1.Esso circonda il malleolo aderendo alla corticale ossea, prima sotto e anteriormente al peroniero lungo, per poi andare ad inserirsi alla base del $\mathrm{V}^{\circ}$ metatarso. Un piccolo fascio può inserirsi alla base del $\mathrm{V}^{\circ}$ metatarso e un prolungamento fino alla base del $\mathrm{V}^{\circ} \mathrm{o}$ unirsi alla base del suo estensore.Il peroniero breve abduce e ruota lateralmente il piede (eversore), antagonista del tibiale posteriore, ma agonista con questo nella flessione La patologia infiammatoria più frequente del tendine peroniero breve si osserva negli atleti che ritornano allo sport dopo un lungo periodo di inattività. Tra le principali cause del dolore nella regione del retro piede c'è la sindrome da over use (uso eccessivo) e malattie sottostanti (artrite reumatoide, diabete mellito). Tali patologie predispongono alla rottura del tendine, che possono essere parziali o totali. Esse possono essere presenti sia nei giovani che negli anziani e sono spesso un reperto accidentale. Negli atleti di solito è sintomatica, e associata a sport che richiedono cambi improvvisi di direzione, come calcio, tennis e basket.

\section{DESCRIZIONE DEL CASO}

Paziente di anni 23, accede al presidio ambulatoriale radiologico con richiesta di approfondimento diagnostico al piede destro, con inquadramento clinico del medico di medicina generale di sospetto sperone calcaneare.

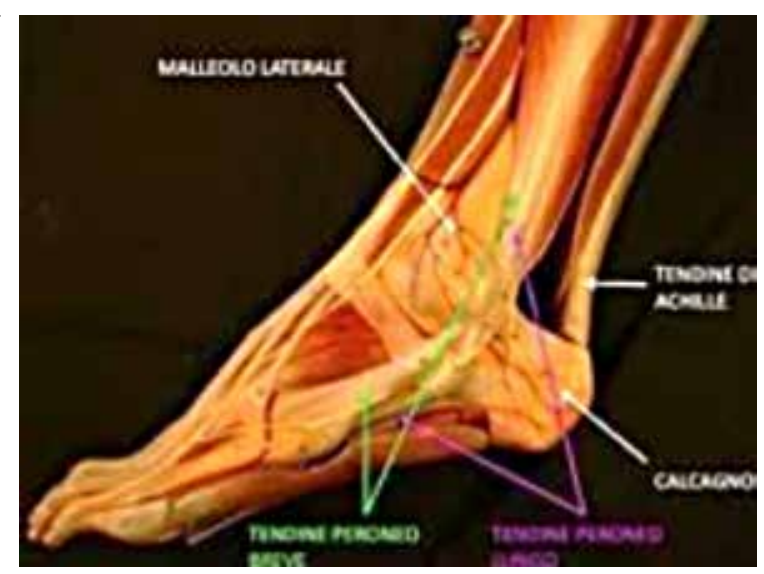

Figura 1. Richiami anatomici, il tendine peroneo breve. Immagine originaria dal sito:http://www.footsurgery.center/instabilita-dei-tendini-peronieri/

Nel posizionarsi sul lettino radiologico, la paziente si avviava verso di esso, assumendo un atteggiamento posturale scorretto con preponderante compensazione sul lato mediale del piede destro. Dopo domanda sulla ragione dell'indagine radiologica, riferiva di un'importante artralgia ingravescente presente da circa sette mesi, presentatasi improvvisamente e senza aver subito traumi della parte in esame. La paziente riferiva inoltre di una forte tensione che si protraeva dalla zona plantare e si diffondeva al sistema muscolare della gamba. Fin dall'insorgenza della sintomatologia dolorosa aveva sempre portato delle calzature piatte, conosciute comunemente come "converse", senza cambio o alternanza dalle stesse. Il forte dolore, con caratteristiche ormai croniche, si contraddistingueva per impedimento funzionale nei movimenti di flesso-estensione del complesso articolare piede-caviglia, con problemi maggiori in carico. La giovane paziente lamentava inoltre la presenza recente e simultanea di dolore, nelle settimane 


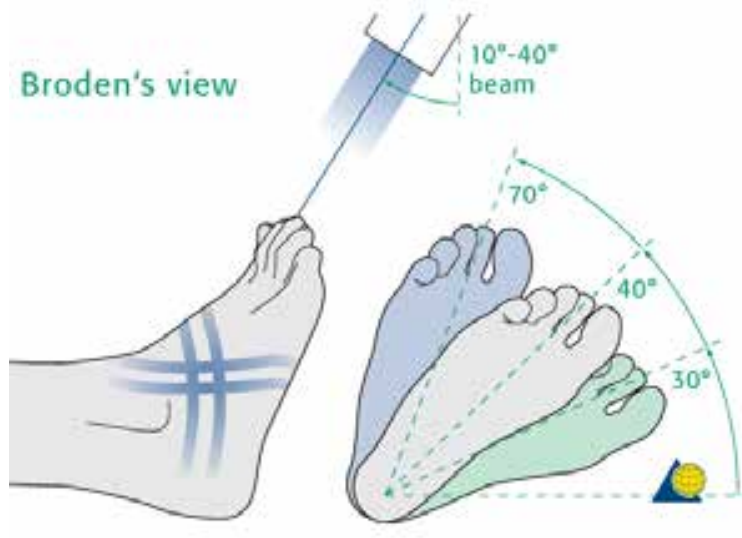

Immagine originaria dal sito: https://www2.aofoundation.org

subito antecedenti l'esame, anche al piede contro laterale. L'accertamento eseguito presso la struttura ambulatoriale è stato il primo e unico effettuato.

\section{MATERIALI E MIETODI}

Coerentemente con il quesito di sospetto sperone calcaneare, è stata eseguita come prima proiezione una latero-laterale del piede destro; l'indagine è proseguita effettuando una proiezione dorso-plantare del piede. Con il consulto del medico radiologo, in riferimento alla storia anamnestica della paziente, si è deciso inoltre da un punto di vista clinico e tecnico di proseguire l'indagine con una proiezione obliqua di caviglia.

Durante il posizionamento, in linea con il quadro su descritto, si è fatto in modo di porre l'articolazione piedecaviglia con un angolo di circa $45^{\circ}$ rispetto al piano d'appoggio e raggio incidente obliquo di circa $10^{\circ}$ in direzione caudo-craniale sui malleoli. La collimazione, sia in senso supero-inferiore che medio-laterale e latero-mediale, è stata ampliata al fine di valorizzare il comparto articolare della caviglia e parte dell'articolazione di Chopart, ma includendo la base del $\mathrm{V}^{\circ}$ metatarso. Il motivo di questa scelta risiede nelle peculiarità offerte dai criteri di correttezza permessi dalla proiezione radiografica di Broden, usata appunto in questa indagine.

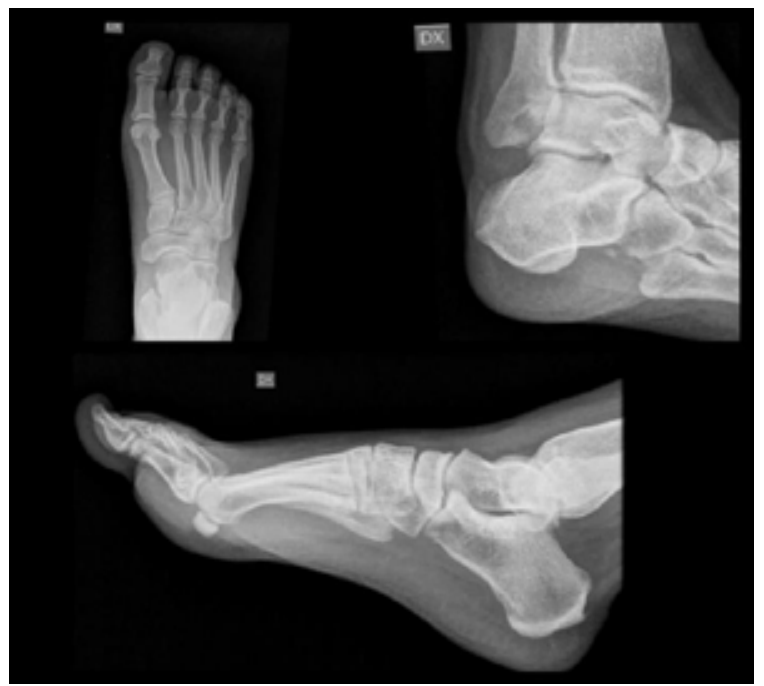

Figura 2. Proiezione dorso-plantare e proiezione obliqua in alto, mentre in basso la proiezione laterale del piede.
L'immagine radiologica, visibile in figura 3, permette non soltanto una chiara visione dell'articolazione talocalcaneare, ma anche di evidenziare la zona anatomica sottesa tra la base del $\mathrm{V}^{\circ}$ metatarso e la tuberosità calcaneare, sede dell'infiammazione cronica.

Nell'immagine sottostante, figura 3, possiamo osservare un quadro riferito alla proiezione obliqua eseguita.

\section{COMIMENTO E ANALISI}

\section{DEILIE IMIMA GINI}

Dall'indagine eseguita non si denotano evidenti alterazioni osteo - strutturali a carico dei distretti scheletrici ed articolari presi in esame. Tuttavia possiamo evidenziare la presenza di formazioni radiopache, dotate di densità calcifica, in grado di proiettare al livello dei tessuti superficiali para- articolari situati nella sede articolare cuboido-calcaneare e metatarso-cuboidea. Tali formazioni calcifiche si dimostrano disposte lungo il decorso del tendine peroneo breve. Grazie al confronto tra l'immagine obliqua in positivo e quella in negativo, si apprezza nella porzione di tale tendine (nella parte più laterale) piccole lacerazioni verosimilmente dovute a piccoli traumi cronicizzati. Il quadro radiografico descritto è stato confermato e ribadito da un ulteriore esame ecografico al quale si è sottoposta la paziente.

\section{RISULTATI}

Nel caso in esame di questa giovane paziente, si porta alla luce un raro caso di diagnosi di tendinite e parziale lacerazione del tendine peroneo breve nella sua porzione metatarsale. Dopo un ascolto attivo del TSRM nei confronti della paziente, è stato possibile individuare la corretta direzione in cui obliquare l'articolazione in esame e il tubo radiogeno, tale da riuscire a proiettare chiaramente la porzione tendinea patologica nel radiogramma.

L'ispessimento tendineo ha infatti determinato un aumento della capacità di attenuazione, rivelando cosi la patologia infiammatoria muscolo scheletrica anche in radiologia convenzionale. La scelta, in sinergia con il medico radiologo, di proseguire l'indagine considerando le caratteristiche biomeccaniche del tendine peroneo breve, l'anamnesi di dolore ingravescente presente

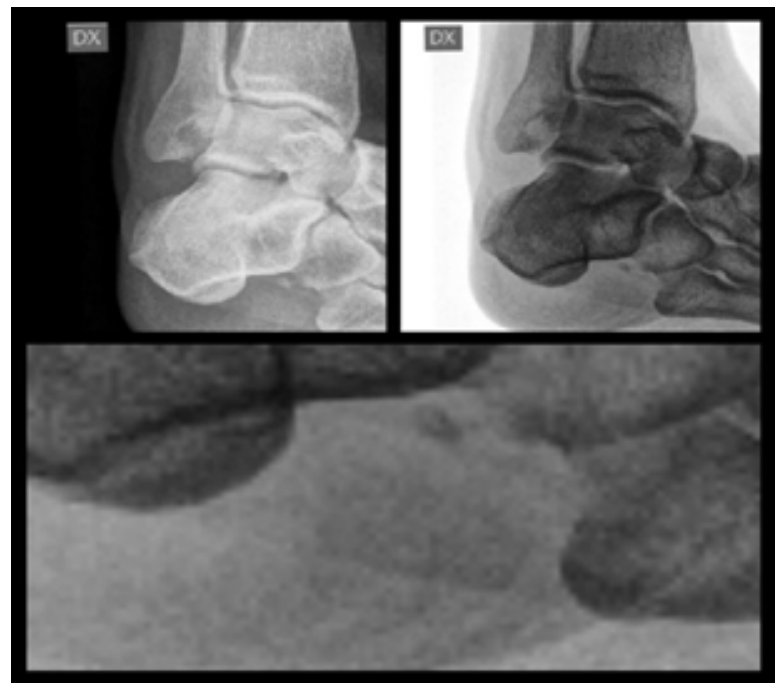

Figura 3. Proiezione obliqua di caviglia. In alto la proiezione obliqua, in positivo a sx e in negativo a dx. Nell'immagine sottostante è disponibile un ingrandimento della stessa. 

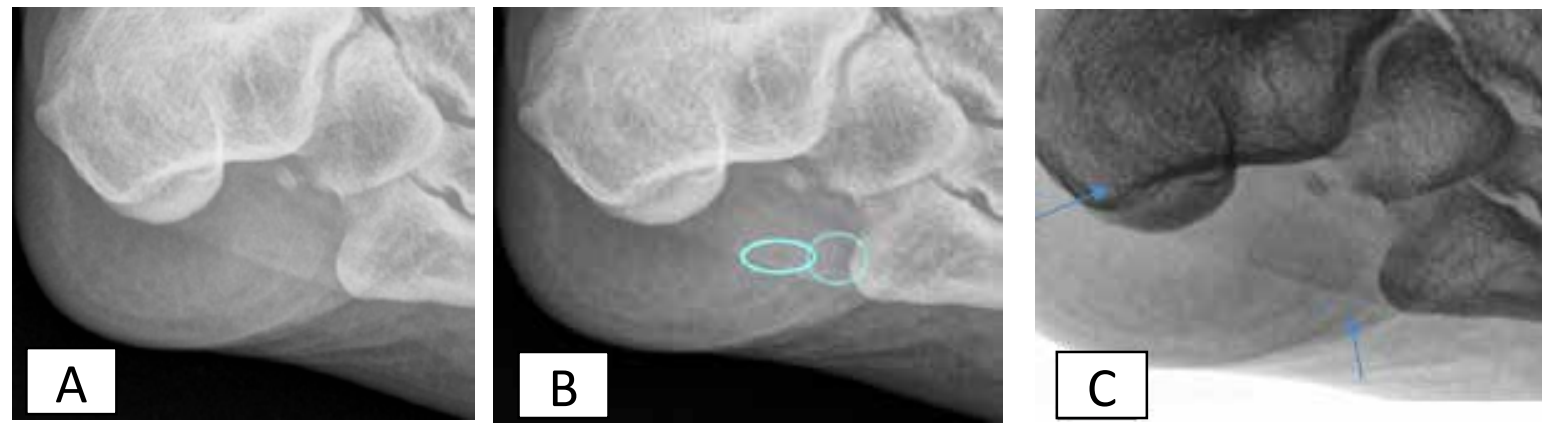

Figura 4. In ordine da sinistra a destra: A. ingrandimento della proiezione obliqua; B. ingrandimento ove è possibile osservare le lacerazioni tendinee nell'intorno dei corpi calcifici. Inoltre possiamo notare nelle zone cerchiate le lacerazioni appartenenti al sito d'inserzione, in particolare quella inscritta nel cerchio più grande. C. Ingrandimento. con il contributo del negativo si riescono a apprezzare meglio le lacerazioni presenti. che appaiono come evidenti radiotrasparenze nel fascio tendineo danneggiato.

già diversi mesi, ha consentito di perfezionare lo studio palesando il sospetto clinico inziale, rafforzato inoltre da un cambiamento evidente della volta plantare.

È importante sottolineare che la giusta tecnica esposimetrica ha determinato un ottimo livello di risoluzione spaziale e di contrasto. L'approccio espositivo è stato fondamentale infatti nel determinare il risultato iconografico: è stata utilizzata una tecnica a due punti data da $43 \mathrm{Kv}$ e $7 \mathrm{mAs}$, con una DFF di circa $115 \mathrm{~cm}$, al fine di favorire la maggiore attenuazione e rivelando un maggior contenuto diagnostico, evidenziando infatti sia il tessuto osseo che quello molle. Inoltre il contributo della versione "negativa" dell'immagine obliqua ha determinato un aumento della nitidezza, nella visualizzazione delle parziali lacerazioni tendinee.

Con questo report si dimostra quanto sia importante la corretta tecnica espositiva e un giusto posizionamento, in base alla biomeccanica dell'articolazione di studio. Grazie infatti a una tecnica ragionata sulla base biomeccanica del distretto piede-caviglia, e all'ascolto della storia anamnestica, è stato possibile capire quanto inclinare l'articolazione per riuscire a ottenere un buon risultato diagnostico.

\section{CONCLUSTIONI}

Con questo report si vuole dimostrare come la figura del Tecnico sanitario di radiologia medica, sia dirimente nella rivelazione di patologie che normalmente non vengono facilmente diagnosticate.

E' sicuramente fondamentale la sinergia con le altre figure mediche e paramediche, ma è ancora più importante che di ciascuna professione vengano valorizzate le caratteristiche peculiari, dando spazio alla libertà di sapere e poter procedere sulla base delle proprie conoscenze e caratteristiche. Nonostante la legge 746/1994 veda vincolato il TSRM a una subordinazione totale al Medico specialista, il tecnico di radiologia libero di poter fare e creare diventa esso stesso produttore di innovazione e apportatore di ricchezza scientifica, grazie all'evidenza diagnostica che si rivela poi nelle sue sperimentazioni.

La preparazione del TSRM dimostra infatti quanto sia importante la corretta tecnica espositiva e un giusto posizionamento, sulla base delle conoscenze tecniche radiologiche, e le conoscenze biomeccaniche dell'articolazione di studio.

Lo studio dei tessuti molli è stato possibile grazie alla coesistenza di diverse variabili, e alla messa in atto di specifiche tecniche di esame tali da rilevare la patologia del piede esistente. Una importante variabile è l'ispessimento tendineo presente che ha generato un aumento della capacità di attenuazione, determinando la visibilità del segmento di tessuto molle in studio anche in radiologia convenzionale.

Grazie a una tecnica ragionata e all'ascolto attivo della storia anamnestica è stato possibile capire precisamente come condurre l'esame, e di conseguenza quanto inclinare l'articolazione per riuscire a ottenere un buon risultato diagnostico.

Il bagaglio culturale ed esperienziale del tecnico sanitario di radiologia medica può quindi fare realmente la differenza, determinando la giusta tecnica d'esame e quindi il giusto inquadramento clinico del paziente.

\section{BIBLIOGRAFIA}

1. Ecografia Patologica Muscoloscheletrica, Testo e Atlante, Galletti - Casa editrice PICCIN

2. Anatomia funzionale II arto inferiore A.I. KAPANDJI $6^{\circ}$ EDIZIONE MALONE - MONDUZZI EDITORE

3. https://www.ncbi.nlm.nih.gov/pubmed/11831128

4. Ecografia Patologica Muscoloscheletrica, Testo e Atlante, Galletti - Casa editrice PICCIN

5. Figura 1: Immagine originaria dal sito:http://www.footsurgery.center/instabilita-dei-tendini-peronieri/

6. Questo report dal titolo "Proiezione obliqua del piede, in radiologia convenzionale, nella diagnosi di tendinite del peroniero breve" è collegato cronologicamente e come percorso diagnostico al report: "L'arco plantare e l'efficienza del peroneo breve". Grazie al primo report abbiamo potuto dare i natali al secondo report. Per quest'ultimo si vuole ringraziare sentitamente per l'importante supporto dato "BERTOLUCCI TECNICA ORTOPEDICA PISTOIESE" sita in Viale Frosini Attilio, 28, 51100 Pistoia. 